recipient: a case study. Clin Infect Dis. 2021;ciab072. https://doi.org/10.1093/cid/ciab072

6. Maggi F, Novazzi F, Genoni A, Baj A, Spezia PG, Focosi D, et al. Imported SARS-COV-2 variant P.1 detected in traveler returning from Brazil to Italy. Emerg Infect Dis. 2021;27:1249-51. https:/ / doi.org/10.3201/eid2704.210183

7. European Medicines Agency. Assessment report Eli Lilly. Company Limited use of bamlanivimab and etesevimab for the treatment of COVID-19, 2021 [cited 2021 Jun 2]. https://www.ema.europa.eu/en/documents/referral/ eli-lilly-company-limited-antibody-combinationbamlanivimab/etesevimab-covid19-article-53-procedureassessment-report_en.pdf

8. Starr TN, Greaney AJ, Dingens AS, Bloom JD. Complete map of SARS-CoV-2 RBD mutations that escape the monoclonal antibody LY-CoV555 and its cocktail with LY-CoV016. Cell Rep Med. 2021;2:100255. https:/ / doi.org/ 10.1016/j.xcrm.2021.100255

Address for correspondence: Andreina Baj, University of Insubria, Via Dunant 5, Varese 21100, Italy; email: andreina.baj@uninsubria.it

\section{Indoor and Outdoor Rodent Hosts of Orientia tsutsugamushi, Shandong Province, China}

\author{
Fei Li, Zhen-Tang Zhang, Li-Zhu Fang, Hao Yu, \\ Xiang-Rong Qin, Xue-Jie Yu
}

Author affiliations: Shandong University, Jinan, China (F. Li); Huangdao District Center for Disease Control and Prevention, Qingdao, China (Z.-T. Zhang); Wuhan University, Wuhan, China (L.-Z. Fang, H. Yu, X.-J. Yu); The Second Hospital of Shandong University, Jinan (X.-R. Qin)

DOI: https://doi.org/10.3201/eid2710.210393

During December 2012-July 2016, we tested small indoor and outdoor mammals in Qingdao, China, for Orientia tsutsugamushi infection. We found that outdoor Apodemus agrarius mice, Cricetulus barabensis hamsters, and Niviventer confucianus rats, as well as indoor Mus musculus mice, tested positive for O. tsutsugamushi by PCR.

Crub typhus is an emerging infectious disease Scaused by Orientia tsutsugamushi (1), which is transmitted through the bites of infected chiggers, the larvae of trombiculid mites of the genus Leptotrombidium. Scrub typhus has been documented in southern China for thousands of years (2) and emerged in northern China during the 1990s (3). Several studies have investigated the animal hosts of $O$. tsutsugamushi $(4,5)$, but the major hosts and seasonality of $O$. tsutsugamushi in northern China remain unclear. We collected small animals in Qingdao, a city in eastern China, to investigate the hosts and seasonality of $O$. tsutsugamushi.

During December 2012-July 2016, we used indoor and outdoor mousetraps to capture 162 small mammals (154 rodents and 8 shrews) in 2 villages in Huangdao District, Qingdao $\left(119^{\circ} 30^{\prime}-121^{\circ} 00^{\prime} \mathrm{E}\right.$, $35^{\circ} 35^{\prime}-37^{\circ} 09^{\prime} \mathrm{N}$ ) (Figure). All animal samples were obtained in accordance with the Implementation Regulations of the People's Republic of China on the Protection of Terrestrial Wild Animals (http:/ / www. gov.cn/zhengce/2020-12/25/content_5574749.htm). The collection of rodents for microbiological studies was approved by the Ethics Committee of Prevention Medicine of Shandong University (Jinan, China; approval no. 20150501).

We classified samples by morphologic characteristics. We captured all 7 Cricetulus barabensis hamsters, 18 Tscherskia triton hamsters, and 8 Niviventer confucianus rats in the fields, as well as $98.6 \%(69 / 70)$ of Apodemus agrarius mice. We captured most Rattus norvegicus rats $(22 / 24 ; 92 \%)$ and Mus musculus mice $(18 / 27 ; 67 \%)$ in indoor settings, as well as $25 \%(2 / 8)$ of Crocidura lasiura shrews.

We extracted and screened DNA from rodent spleens for O. tsutsugamushi by nested PCR selective for the 56-kDa type-specific antigen gene with outer primers (5'- TCAAGCTTATTGCTAGTGCAATGTCTGC-3' and 5'-AGGGATCCCTGCTGCTGTGCTTGCTGCG-3') and inner primers (5'-GATCAAGCTTCCTCAGCCTACTATAATGCC-3' and 5'-CTAGGGATCCCGACAGATGCACTATTAGGC-3') $(6,7)$. Overall, $4.5 \%$ of 154 rodents but none of the 8 shrews were positive for $O$. tsutsugamushi.

All infected rodents were captured during autumn (i.e., September-November); among rodents captured in autumn, the infection rate was $8.1 \%$ (7/86; p $>0.05$ by 1 -sided Fisher exact test). None of the 68 rodents captured during spring, summer, and winter tested positive for O. tsutsugamushi (Table). The absence of O. tsutsugamushi infection among rodents collected during spring, summer, and winter indicated that these rodents were not reservoirs but temporary amplifying hosts for O. tsutsugamushi. The presence of $O$. tsutsugamushi among rodents during autumn months is consistent with the seasonality of 


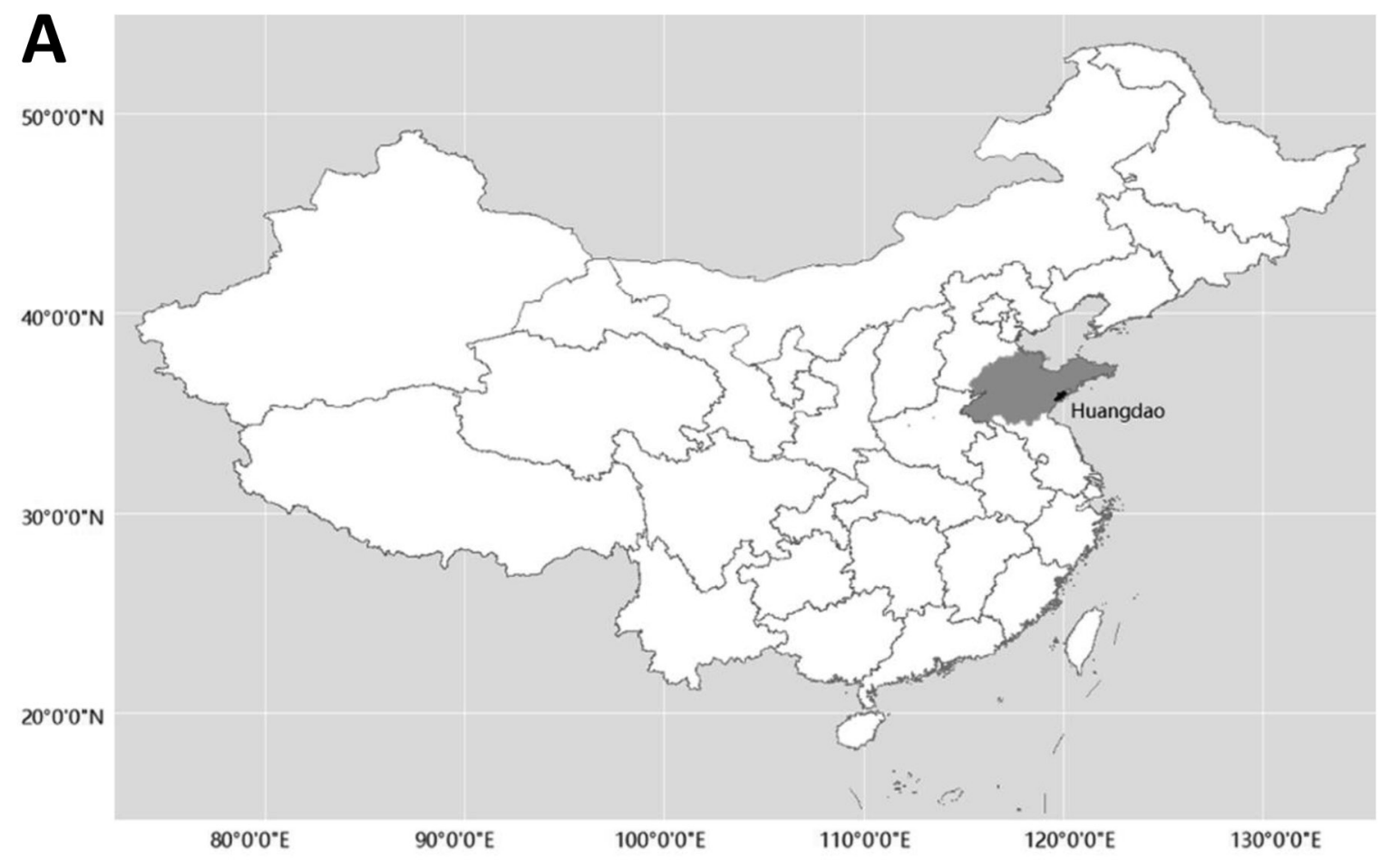

B

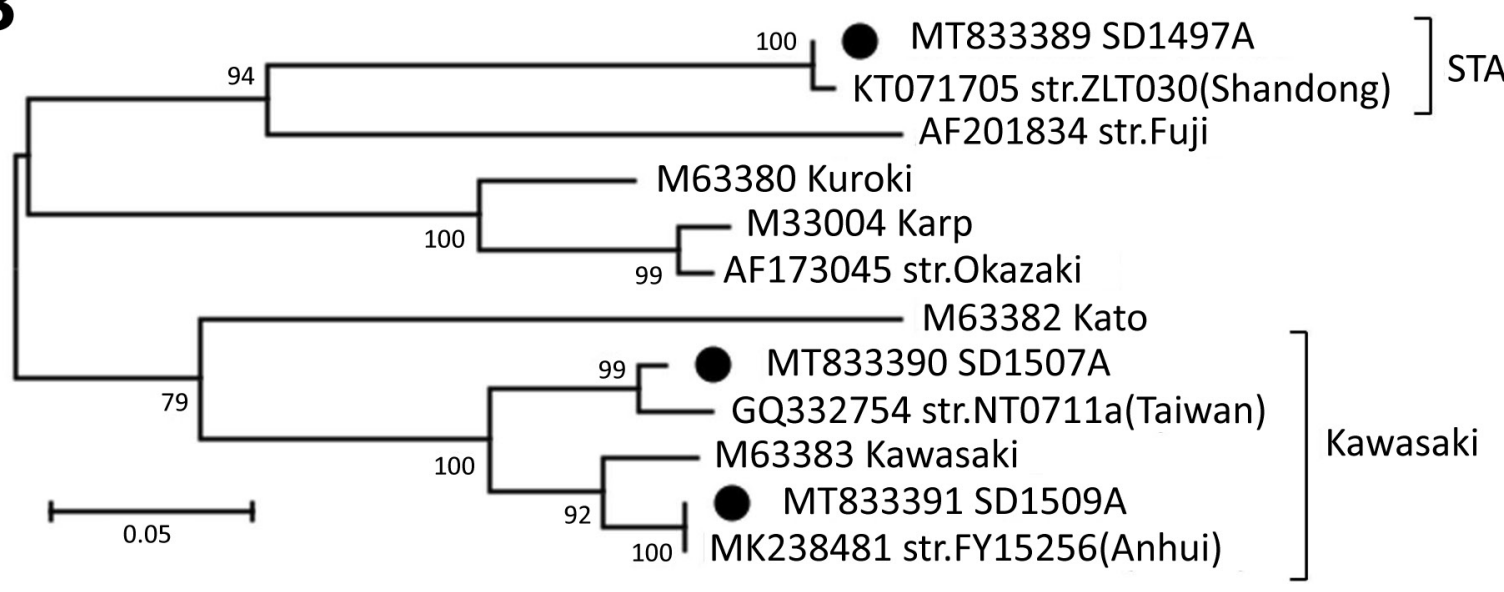

Figure. Orientia tsutsugamushi in small mammals, Qingdao, Shandong Province, China, December 2012-July 2016. A) Location of Shandong Province in China. B) Maximum-likelihood phylogenetic tree of O. tsutsugamushi constructed by MEGA version 7.0 (http:// www.megasoftware.net). Black circles indicate strains isolated in this study. Numbers to the left of nodes indicate bootstrap values based on 1,000 replicates. Scale bar indicates number of nucleotide substitutions per site.

scrub typhus among patients in Shandong Province. Among humans, O. tsutsugamushi infections occur during September-December and peak in October (8), in alignment with the reproductive season of Leptotrombidium scutellare mites (3).

Overall, we found that $4.3 \%$ of A. agrarius mice, $7.4 \%$ of $M$. musculus mice, $12.5 \%$ of $N$. confucianus rats, and $14.3 \%$ of $C$. barabensis hamsters tested positive for O. tsutsugamushi; these results indicate the potential role of these rodents as animal hosts for O. tsutsuga- mushi. None of the $24 R$. norvegicus rats, $18 \mathrm{~T}$. triton hamsters, or $8 \mathrm{C}$. lasiura shrews tested positive, suggesting that these animals are not major hosts for $O$. tsutsugamushi. $R$. norvegicus rats, which were mainly captured indoors, were all negative for $O$. tsutsugamushi; these findings suggest that these rats might primarily stay indoors, thereby avoiding exposure to chiggers in the fields. We found $2(11.1 \%)$ O. tsutsugamushi-positive indoor house mice, possibly reflecting their travels between house and field. 
Table. Prevalence of Orientia tsutsugamushi in small mammals, Qingdao, Shandong Province, China, December $2012-J u l y ~ 2016$

\begin{tabular}{lccccc}
\hline & \multicolumn{4}{c}{ Season, no. positive/no. tested (\%) } \\
\cline { 2 - 6 } Species & Spring & Summer & Autumn & Winter & Total \\
\hline Rodent & & & & & \\
$\quad$ Apodemus agrarius & $0 / 2(0)$ & $0 / 28(0)$ & $3 / 39(7.7)$ & $0 / 1(0)$ & $3 / 70(4.3)$ \\
$\quad$ Mus musculus & $0 / 2(0)$ & $0 / 14(0)$ & $2 / 9(22.2)$ & $0 / 2(0)$ & $2 / 27(7.4)$ \\
$\quad$ Rattus norvegicus & $0 / 4(0)$ & $0 / 8(0)$ & $0 / 9(0)$ & $0 / 3(0)$ & $0 / 24(0)$ \\
$\quad$ Tscherskia triton & $0 / 1(0)$ & $0 / 1(0)$ & $0 / 15(0)$ & $0 / 1(0)$ & $0 / 18(0)$ \\
$\quad$ Niviventer confucianus & 0 & 0 & $1 / 8(12.5)$ & 0 & $1 / 8(12.5)$ \\
$\quad$ Cricetulus barabensis & $0 / 1(0)$ & 0 & $1 / 6(16.7)$ & 0 & $1 / 7(14.3)$ \\
\hline Shrew & 0 & 0 & $0 / 8(0)$ & 0 & $0 / 8(0)$ \\
$\quad$ Crocidura lasiura & 0 & $0 / 51(0)$ & $7 / 94(7.4)$ & $0 / 7(0)$ & $7 / 162(4.3)$ \\
\hline Total & $0 / 10(0)$ & & &
\end{tabular}

The sequences of $O$. tsutsugamushi from rodents identified belonged to 2 lineages, Kawasaki and STA-07 (Figure). We identified the Kawasaki strain in 4 rodent species collected in the same village during the autumns of 2013 and 2014 and the STA-07 strain in A. agrarius mice in a village $20 \mathrm{~km}$ away during the autumn of 2014. These results suggest that $O$. tsutsugamushi isolates from same geographic area are highly homologous regardless of host species. We deposited the 56-kDa type-specific antigen gene sequences obtained in this study in GenBank (accession nos. MT833389-95).

In conclusion, we documented $O$. tsutsugamushi infection among outdoor A. agrarius mice, N. confucianus rats, and C. barabensis hamsters, as well as indoor M. musculus mice, in Shandong Province; these rodents might serve as animal hosts for O. tsutsugamushi. The finding of $O$. tsutsugamushi infection among indoor mice suggest that persons might be exposed to chiggers and O. tsutsugamushi at home. Further study is needed to investigate whether scrub typhus patients in the area had a history of working or traveling in the fields and whether their houses were infested with mice and chiggers. Our results indicate that physicians should be attentive to patients who might have O. tsutsugamushi infection, even if those patients have not worked in the field.

\section{About the Author}

Ms. Li is a doctoral candidate at Shandong University, Jinan, China. Her research interests include emerging infectious disease and vector-borne disease.

\section{References}

1. Walker DH. Rickettsiae. In: Baron S, editor. Medical Microbiology. 4th ed. Galveston (TX): University of Texas Medical Branch at Galveston; 1996.

2. Fan MY, Walker DH, Yu SR, Liu QH. Epidemiology and ecology of rickettsial diseases in the People's Republic of China. Rev Infect Dis. 1987;9:823-40. https:/ / doi.org/ 10.1093/clinids/9.4.823

3. Zhang S, Song H, Liu Y, Li Q, Wang Y, Wu J, et al. Scrub typhus in previously unrecognized areas of endemicity in China. J Clin Microbiol. 2010;48:1241-4. https:/ / doi.org/ 10.1128/JCM.01784-09

4. Liu YX, Jia N, Xing YB, Suo JJ, Du MM, Jia N, et al. Consistency of the key genotypes of Orientia tsutsugamushi in scrub typhus patients, rodents, and chiggers from a new endemic focus of northern China. Cell Biochem Biophys. 2013;67:1461-6. https:/ / doi.org/10.1007/s12013-013-9646-0

5. Wu G-H,Wang C-J, Li B-J, Jiang Z-K, Ding L-Y,Wang L. General situation on studies of animal hosts of tsutsugamushi disease in China. Chinese Journal of Hygienic Insecticides of Equipments. 2013;19:370-3.

6. Tamura A, Yamamoto N, Koyama S, Makisaka Y, Takahashi M, Urabe K, et al. Epidemiological survey of Orientia tsutsugamushi distribution in field rodents in Saitama Prefecture, Japan, and discovery of a new type. Microbiol Immunol. 2001;45:439-46. https://doi.org/10.1111/j.1348-0421.2001.tb02643.x

7. Furuya Y, Yoshida Y, Katayama T, Yamamoto S, Kawamura A Jr. Serotype-specific amplification of Rickettsia tsutsugamushi DNA by nested polymerase chain reaction. J Clin Microbiol. 1993;31:1637-40. https:/ / doi.org/10.1128/ jcm.31.6.1637-1640.1993

8. Wu GH. The epidemiological characteristics and prevention and cure of scrub typhus in China. China Public Health. 2000;16:777-9.

Address for correspondence: Xiang-rong Qin, 247 Beiyuandajie, Jinan, Shandong Province 250033, China; email:

qinxiangrong07@qq.com; Xue-jie Yu, 115 Donghu Rd, Wuhan, Hubei 430071, China; email: yuxuejie@whu.edu.cn 